\title{
A aplicação do Kanban como ferramenta de gestão em serviços de saúde: revisão integrativa
}

RESUMO | Objetivo: analisar a produção científica recente sobre aplicação da ferramenta Kanban na gestão dos serviços de saúde, valorando as evidências e discutindo os resultados. Método: revisão integrativa de literatura, realizada com artigos publicados entre os anos de 2014 a 2018, disponíveis nas bases digitais: LILACS, PubMed e SciELO. Os dados foram tratados qualitativamente, utilizando a técnica de análise de conteúdo temática. Resultados: foram analisados 13 artigos que apontaram a utilização da ferramenta em três áreas: estoque de medicamento, fluxo de paciente e qualidade de assistência à saúde. Um único artigo, experimental, simulava em laboratório o uso da ferramenta no trabalho diário. Os estudos envolvendo a gestão de leitos não relacionaram kanban ao processo de internação em pediatria. Conclusão: os estudos evidenciaram a aplicabilidade da ferramenta na gestão dos serviços em saúde, reduzindo o tempo de internação, colaborando no processo de insumos hospitalares e agregando qualidade a assistência.

Palavras-chaves: acompanhamento dos cuidados de saúde; gestão de qualidade; gestão em saúde.

ABSTRACT | Objective: to analyze the recent scientific production on the application of the Kanban tool in the management of health services, assessing the evidence and discussing the results. Method: integrative review of literature, using articles published between the years of 2014 and 2018, available in the digital databases: LILACS, PubMed and SciELO. The data were treated qualitatively, applying the thematic content analysis technique. Results: 13 articles that indicated the use of the tool in three areas were analyzed: drug stock, patient flow and health care quality. A single experimental paper simulated in laboratory the use of the tool in daily work. Studies involving bed management did not relate kanban to the process of pediatric hospitalization. Conclusion: the studies revealed the applicability of the tool in the management of health services, reducing hospitalization time, improving hospital supply chain management and adding quality to care.

Keywords: continuity of patient care; quality management; health management.

RESUMEN | Objetivo: analizar la reciente producción científica sobre aplicación de la herramienta Kanban en la gestión de los servicios de salud, valorando las evidencias y discutiendo los resultados. Método: revisión integradora de literatura, realizada con artículos publicados entre los años 2014 a 2018, disponibles en las bases digitales: LILACS, PubMed y SciELO. Los datos fueron tratados cualitativamente, utilizando la técnica de análisis de contenido temático. Resultados: se analizaron 13 artículos que apuntaron la utilización de la herramienta en tres áreas: stock de medicamento, flujo de paciente y calidad de asistencia a la salud. Un único artículo, experimental, simulaba en laboratorio el uso de la herramienta en el trabajo diario. Los estudios involucrando la gestión de lechos no relacionaron kanban al proceso de internación en pediatría. Conclusión: los estudios evidenciaron la aplicabilidad de la herramienta en la gestión de los servicios en salud, reduciendo el tiempo de internación, colaborando en el proceso de insumos hospitalarios y agregando calidad a la asistencia.

Palabras claves: continuidad de la atención al paciente; gestión de la calidad; gestión en salud.

\section{Corina Maria Mattos}

Graduada em Enfermagem e Obstetrícia com Hab. em Saúde Pública (EEAP/UNI-RIO); Especialista nas áreas de: QSMS (UCB/RJ), preceptoria no SUS (IEP/HSL/SP) e gestão em Ativação de Processos (FIO(RUZ), Me. em Enfermagem Assistencial (MPEA/UFF); Enfa. do NSP (HIIS/UPA IWG/ SMSDC. Membro do NECIGEN

\section{Marcia Farias de Oliveira}

Enfermeira com Hab. Obstétrica (EEAN/ UFRJ). Residência em Pediatria (UERJ). Especialista em Neonatologia (IFF/FIOCRUZ). Plantonista no HIIS/SMSDC. Enfermeira Intensivista no Núcleo Perinatal (HUPE/ UERJ). Me. em Ciências do Cuidado em Saúde (PACCS/UFF). Membro do NECIGEN (EEAAC/UFF)

\section{Andrea Maria Alves Vilar}

Graduada em Enfermagem e Obstetrícia com Hab. Médico Cirúrgico (EEAP/UNI-RIO). Residência em Pediatria Intensivista (IFF/FIOCRUZ). Especialista nas áreas: Auditoria em Serviços de Saúde (UES/RJ) e Controle do Câncer (INCA). Enfa. Plantonista da UTI Neonatal no HUPE(UERJ)e do CC e RPA no INCA(MS). Me. em Saúde Materno Infantil (PACCS/UFF)

Recebido em: 18/02/2019

Aprovado em: 13/05/2019

\section{Zenith Rosa Silvino}

Enfermeira Obstétrica (EEAAC/UFF). Licenciatura Plena em Enfermagem (UFF). Me. em Direito (UGF/RJ). Dra. em Enfermagem (UFRJ) e Pósdoutorado (UERJ). Prof. Tit. na área de Administração (UFF). Avaliadora Institucional e de Cursos do INEP. Membro Tit. da ABAH. Coord. Adj. da REPEGENF

\footnotetext{
Marilda Andrade

Enfermeira (EEAN/UFRJ). Especialista em Adm. Hospitalar (UERJ) Me. em Enfermagem (EEAN/UFRJ). Dra. em Enfermagem (UFRJ). Vice-diretora e Prof. Assoc. IV da EEAAC/UFF. Membro do Conselho da Rev. Online Brazilian Journal. Membro do NEPGTS
} 


\section{INTRODUÇÃO}

$\mathrm{K}$ anban é uma palavra japonesa que significa, literalmente, cartão. Nos anos pós-segunda guerra mundial, passou a ser utilizada na indústria japonesa para designar técnica de controle de solicitação de componentes entre equipes da mesma linha de produção, utilizando placas, cartões ou outro sinal visível ${ }^{1-2}$.

A ferramenta Kanban foi desenvolvida para dar suporte ao Sistema Toyota de Produção (STP), método de produção automobilística da Toyota Motor Company. Idealizada por Tiichi Ohno na década de 50, consistia em uma proposta de instrumento de controle para os processos de fabricação em série denominados just in time (JIT). Aplicada nas etapas de aprovisionamentos, produção e distribuição deste método de gerir existências, proporcionaria suporte e nivelamento da produção, com minimização dos estoques de produtos intermediários e finais ${ }^{1,3}$. Em 2012 são publicados no Brasil os primeiros trabalhos científicos sobre adaptação da ferramenta Kanban em serviços de urgência e emergência, no âmbito do SUS, com vistas a atender demandas específicas deste setor ${ }^{6}$. A desproporção crescente entre o número de leitos de internação oferecidos e a necessidade real dos usuários evidenciou não só a necessidade da expansão de oferta de leitos na rede pública como também a importância da gestão dos mesmos, com monitoramento do tempo de permanência, um indicador importante que possibilita medir a eficiência, eficácia e efetividade da gestão, e influencia no controle dos custos $^{6-7}$.

Com a finalidade de melhorar o fluxo de pacientes da Unidade de Pronto Atendimento (UPA) pediátrica e controlar o tempo de permanência nos leitos do hospital infantil municipal, a secretaria de saúde do município de Duque de Caxias, em 2014, solicitou às coordenações de enfermagem e médica que formassem grupos de profissionais para viabilizar a implantação da ferramenta kanban nestas unidades.

Esta estratégia foi sugerida pelo $\mathrm{Mi}$ nistério da Saúde (MS) como parte de um projeto que previa a realização de mudanças estruturais e organizacionais em instituições participantes do Programa SOS Emergências ${ }^{8}$. O Kanban foi definido como ferramenta com potencial para dar visibilidade a pacientes com período de internação acima da média aceitável, facilitando a percepção deste problema e contribuindo para sua solução. Como o kanban é um sistema visual, foram utilizadas cores padronizadas para toda a rede: verde, servindo para sinalizar pacientes com tempo de permanência aceitável; amarela, aplicada àqueles no limite de tempo de internação; e vermelho para evidenciar pacientes que ultrapassassem o tempo de internação que o SUS considera aceitável ou limítrofe 9 .

O uso da ferramenta kanban serviria à gestão de leitos hospitalares destas duas unidades, componentes da Rede de Atenção às Urgências e Emergências (RUE), incluídas no sistema online de regulação de leitos, o Sistema Nacional de Regulação de vagas (SISREG), criado para o gerenciamento de todo complexo regulatório, indo da rede básica à internação hospitalar. A criação do SISREG visava a humanização dos serviços de saúde, com maior controle do fluxo e otimização na utilização dos recursos ${ }^{10}$.

A experiência de utilização da metodologia kanban na gestão de leitos hospitalares pediátricos do município de Duque de Caxias foi posteriormente objeto de estudo de dissertação de mestrado, publicada em 2016. A ferramenta se comprovou um elo para a gestão do cuidado ${ }^{11}$. Uma avaliação crítica, baseada nos indicadores de saúde gerados pela implantação do kanban, foi realizada em 2018 pela mesma equipe idealizadora do processo.

Este estudo representa uma contribuição para o SUS ao divulgar evidên- cias científicas que respaldem a aplicação da ferramenta de gestão Kanban na qualificação do gerenciamento do cuidado à saúde.

Neste contexto, teve como objetivo analisar a produção científica recente sobre aplicação da ferramenta Kanban na gestão de serviços de saúde, valorando as evidências e discutindo os resultados apresentados.

A questão de pesquisa a ser respondida foi: quais as evidências científicas dos resultados obtidos com o uso do kanban como ferramenta de gestão nos serviços de saúde?

\section{MÉTODO}

Trata-se de uma Revisão Integrativa da literatura, que tem as seguintes etapas: determinação da questão norteadora da pesquisa; estabelecimento dos critérios de inclusão e exclusão; realização da busca em base de dados; categorização e avaliação dos estudos; discussão dos dados e síntese do conhecimento ${ }^{12}$.

A questão norteadora a ser atendida foi: quais os resultados obtidos com a aplicação da ferramenta Kanban na gestão dos serviços de saúde?

Para coleta de dados foi utilizado um instrumento em que incluía os itens: referência dos autores, títulos do artigo, tipo de estudo, ano de publicação, país de origem do estudo, país de publicação, nome do periódico e base de dados (Quadro 2).

Para investigação dos artigos na literatura realizou-se buscas na base de dados Literatura Latino Americana e do Caribe em Ciências da Saúde (LILACS), no portal de serviço PubMed, especializado em literatura biomédica e área afins, e na biblioteca virtual Scientific Eletronic Library Online (SciELO). Suas etapas foram realizadas entre novembro e dezembro de 2018.

A fim de possibilitar a melhor pesquisa e recuperação de artigos possíveis, visto que os termos "Kanban", "Lean", "metodologia Lean" e "gestão 
enxuta” não são descritores da área da saúde, a alternativa foi empregá-los como palavras-chaves, associando-as a descritores próprios desta área, escolhidos por aproximação ao tema, como "Saúde","Leitos", "Administração Hospitalar" e "Gestão", em suas versões inglesa e portuguesa. Os conectores booleanos "AND", "NOT" e "OR" orientaram as buscas por união/exclusão. As estratégias de buscas variaram para atender as especificidades inerentes a cada base de dado utilizada (Quadro1).

Os critérios de inclusão definidos para seleção dos artigos foram: artigos científicos disponíveis online no formato completo, nos idiomas espanhol, inglês ou português, publicados entre os anos de 2014 e 2018 e contendo em seus títulos e/ou resumos os termos da busca. Como critérios de exclusão foram determinados: artigos publicados em anais de congressos ou portais de

Quadro 1. Demonstrativo da utilização de descritores e palavras-chave por base de dados

LILACS (tw:(Kanban OR lean management" OR "lean methodology"OR "gestão enxuta" OR "manufactura esbelta")) AND (instance:"regional") AND (db: ("LILACS))AND ((year cluster: ("2014"OR "2015"or"2016"OR"2017"OR"2018")

PubMed ((Kanban[tw] OR "lean management"[ti]) OR "lean methodology"[ti]) AND (Hospital management[mh] OR Hospital Bed Capacity[tiab] OR Medical[tial] OR Health[tiab]) AND (2013/11/21"[PDat]:"2018/11/19[PDat])

SciELO (kanban OR "lean management" OR "lean methodology" OR "manufactura esbelta" OR "metodologia lean") AND ("Hospital management" OR "Hospital Bed Capacity" OR hospital* OR medical OR health OR saude) AND year_cluster:("2017" OR "2015" OR "2016" OR "2013" OR "2014" OR "2018")

Fonte: elaboração própria, 2018.

eventos científicos, editoriais e artigos que não respondiam à questão norteadora.

A fase de busca foi realizada por três autoras em dois momentos distintos. No primeiro cada avaliadora acessou uma base de dados, arquivando seus resultados primários no gerenciador EndNote Web®. O segundo mo- mento destinou-se à repetição das três estratégias de buscas e foi realizado coletivamente. Comparando os resultados obtidos nestes dois momentos, foi possível verificar que não houve alteração no $\mathrm{n}$ de artigos capturados. Deste modo, a amostra inicial constitui-se de cinquenta e seis artigos. sendo quatro da LILACS, quarenta e três da PubMed,

\section{Figura 1. Fluxograma de busca de artigos para revisão integrativa. Elaboração própria, 2018}

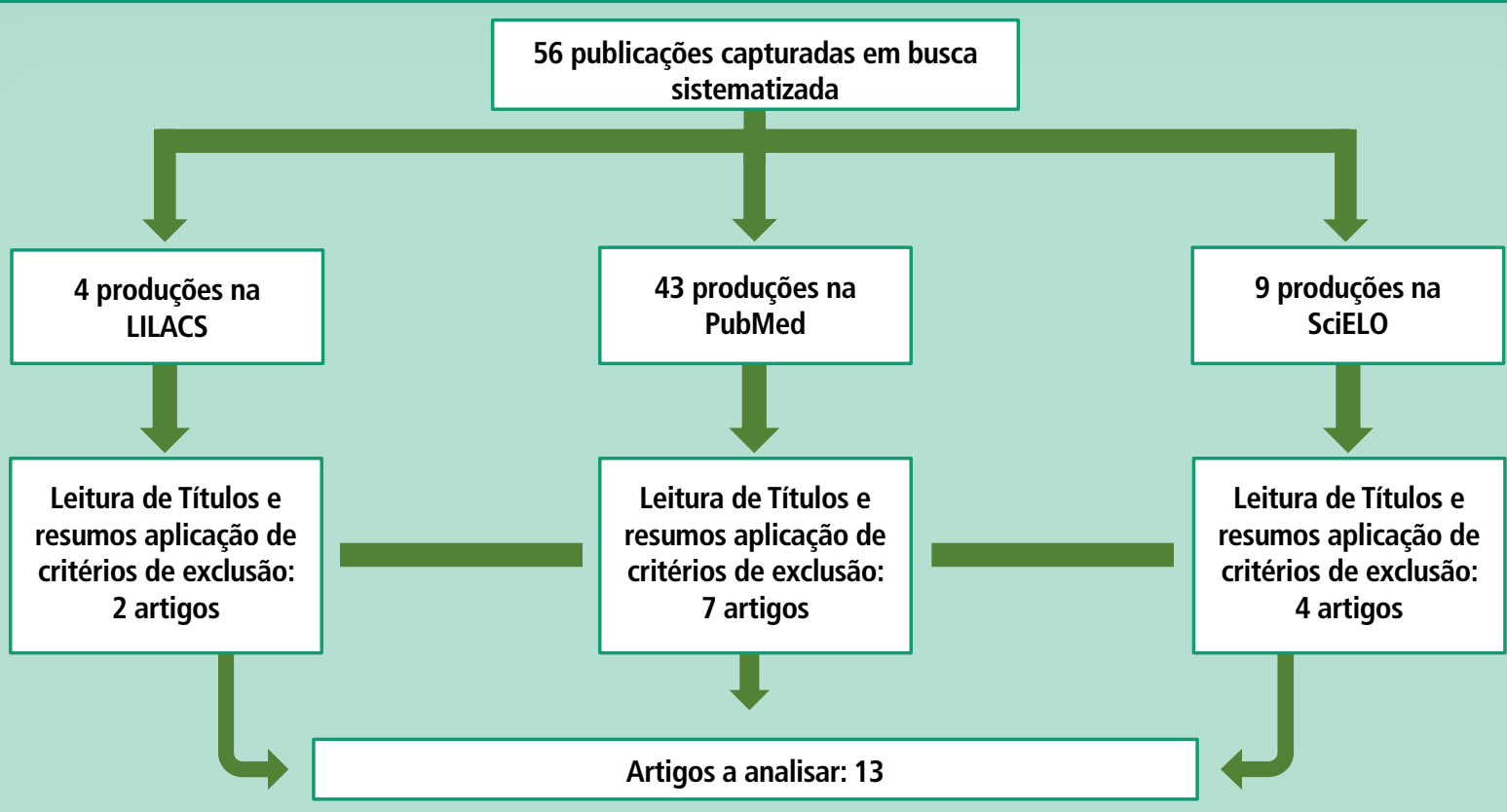

43 publicações excluídas: 2 dissertações, 3 citações sem texto completo disponível, 2 publicações em anais de congressos, 4 artigos revisões sistemáticas, 2 artigos em chinês, 1 artigo na área de educação, e 29 artigos que descreviam a aplicação da metodologia Lean, mas não da ferramenta Kanban. 
e nove da SciELO. Não houve duplicidade de títulos capturados. A leitura dos resumos resultou na exclusão de sete títulos: duas dissertações, três citações sem texto completo disponível e duas publicações em anais de congressos. Dos quarenta e nove artigos elencados para leitura completa, trinta e seis foram excluídos: quatro revisões sistemáticas, dois artigos em chinês, um artigo na área de educação, e vinte e nove artigos que descreviam a aplicação da metodologia Lean, mas não da ferramenta Kanban.

O fluxograma do processo que selecionou os estudos para análise consta na figura 1.

Os dados foram extraídos e dispostos em dois quadros sinópticos, construídos no editor de textos Microsoft Word 2010®. Em um quadro foram agrupados dados para caracterização das produções revisadas. Assim como a classificação por níveis de evidência, possibilitada pelo emprego de uma metodologia estadunidense ${ }^{13}$, que divide hierarquicamente os estudos em seis níveis (Quadro 2). Em outro quadro os estudos foram dispostos e divididos por objetivo, resultados e área temática. (Quadro 3). Cabe ressaltar que agrupamento dos conteúdos foi realizado após verificação das áreas de gestão onde a ferramenta Kanban foi aplicada, servindo à divisão por área temática para análise qualitativa. As categorias temáticas estoque de medicamento, fluxo de paciente e qualidade de assistência à saúde emergiram do processo de análise.

\section{RESULTADOS}

O corpus para análise foi composto por 13 publicações que atenderam aos critérios estabelecidos. Destacamos que uma revisão que expõe uma pequena produção, composta de artigos originais em uma determinada área, pode indicar a necessidade de novos estudos sobre o tema.

Da PubMed foram elencados sete artigos, a maioria $(53,8 \%)$ da amostra. Quatro (30,8\%) oriundos da SciELO e dois $(15,4 \%)$ da LILACS complementaram a amostra. Em relação ao ano de publicação, 2017 foi o de maior expressão, com cinco artigos (38,5\%). Quatro artigos (30,7\%) foram publicados em 2015. De 2018 dois artigos foram analisados (15,4\%). Do ano de 2014 foi avaliado um $(7,7 \%)$ artigo, assim como de 2016. Posicionando os estudos de acordo com os países em que foram realizados, o Brasil se destacou. Ressaltamos que a busca por artigos foi realizada em três bases distintas, que expõem publicações do mundo inteiro. No país foram desenvolvidos sete $(53,8 \%)^{14-17,19,20,23}$ dos treze estudos analisados. Os outros seis $(46,2 \%)$ foram de países diversos: Líbano ${ }^{18}, \mathrm{Co}-$ lômbia ${ }^{21}$, Espanha ${ }^{24}$, Estados Unidos ${ }^{26}$, Grécia $^{22}$ e Suécia ${ }^{25}$. Cinquenta e dois autores participaram da elaboração dos artigos. O artigo da Grécia (7,7\%) foi de autoria individual ${ }^{22}$. Um artigo brasileiro teve oito autores ${ }^{17}$. Dentre os doze periódicos científicos responsáveis pelas publicações, quatro eram brasileiros (33,3\%). Uma destas publicações, a Revista de Administração em Saúde, publicou dois artigos ${ }^{16,20}$. Três periódicos (25\%) eram do Reino Unido (Quadro 2).

A classificação por nível de evidência não identificou estudos do tipo I, II ou IV. Nove estudos (69,2\%) tiveram suas evidências especificadas como nível VI, por se tratarem de estudos qualitativos ${ }^{15-16,19-25}$. Os outros quatro estudos $(30,8 \%)^{14,17-18,26}$ tiveram suas evidências especificadas como nível III, por se tratarem de pesquisas intervencionistas, de campo, bem delineados, sem randomização (Quadro 2). Nível de evidência é critério muito utilizado nas revistas científicas modernas, de padrão internacional. Alguns autores defendem que esta classificação é ferramenta para análise crítica dos trabalhos, orientando a valoração das evidências de pesquisas quanto à aplicabilidade de seus resultados em outros cenários $^{13,27}$. Porém esta aplicabilidade só é possível quando as evidências expostas são fortes. O que não é valido para a maioria $(69,2 \%)$ dos estudos analisados.

Através do processo de análise aplicado, emergiram as categorias temáticas. A categoria qualidade da assistência à saúde, concentrou sete (54\%) artigos. A categoria fluxo de pacientes compreendeu três publicações (23\%). A última, estoque de medicamentos agregou três artigos (23\%) (Quadro 3).

\section{DISCUSSÃO}

Qualidade de assistência a saúde

A qualidade da assistência prestada à saúde é uma preocupação comum entre as instituições que adotam a metodologia Lean nas suas práticas de gestão clínicas e gerenciais ${ }^{17}$, visando principalmente o acompanhamento dos cuidados de saúde e a gestão administrativa dos serviços nas unidades de saúde 17-20,24-26. A Lean Healthcare enfoca e valoriza a satisfação total do cliente, aplicando na saúde ferramentas que visam organizar e gerenciar os serviços tal qual na indústria, em busca da melhoria continua do atendimento prestado para atingir uma gestão de qualidade ${ }^{17-20,24-26}$.

O Kanban é uma das ferramentas da metodologia Lean que tem possibilidade de apresentar informações de forma visual, conforme uma padronização pré-estabelecida 17-20,24-26. Sua aplicabilidade na assistência foi avaliada na área de urgência, emergência e terapia intensiva como uma ferramenta de gerenciamento de qualificação do cuidado que organizou o componente hospitalar ${ }^{20,25}$. Os gestores hospitalares destacaram a ferramenta como um instrumento facilitador de tomada de decisão, estratégico no fornecimento de informações, na criação de indicadores de saúde e redutor de $\operatorname{custos}^{17-18,20,24}$.

Outra qualidade apontada foi a possibilidade oferecida às equipes mul- 


\begin{tabular}{|c|c|c|c|c|}
\hline $\begin{array}{l}\mathrm{N}^{\circ} \text { ref. } \\
\text { Autores }\end{array}$ & $\begin{array}{l}\text { Título } \\
\text { Tipo de estudo }\end{array}$ & $\begin{array}{l}\text { Ano } \\
\text { País Origem/Publicação }\end{array}$ & $\begin{array}{l}\text { Periódico } \\
\text { Base de dados }\end{array}$ & N. E \\
\hline $\begin{array}{l}\text { 14. Rocha; Santos; } \\
\text { IAlcântara; Lima; } \\
\text { Cardoso; Cremonin }\end{array}$ & $\begin{array}{l}\text { Bed Management Tean With Kanban Web-Based Aplication } \\
\text { Intervencionista de campo, quantitativo }\end{array}$ & $\begin{array}{l}2018 \\
\text { BRA } \\
\text { GBR }\end{array}$ & $\begin{array}{l}\text { Int J Qual Heal- } \\
\text { th Car } \\
\text { LILACS }\end{array}$ & III \\
\hline $\begin{array}{l}\text { 15.Lot; Min;Boin; Sa- } \\
\text { rantopoulos; Perales; } \\
\text { Ataide }\end{array}$ & $\begin{array}{l}\text { Using Lean Tools To Reduce Patiente Waiting Time } \\
\text { Qualitativo, de pesquisa-ação }\end{array}$ & $\begin{array}{l}2018 \\
\text { BRA } \\
\text { GBR }\end{array}$ & $\begin{array}{l}\text { Leardsh Healty } \\
\text { Serv } \\
\text { PUBMED }\end{array}$ & $\mathrm{VI}$ \\
\hline $\begin{array}{l}\text { 16. Fuccia; Matsufugi; } \\
\text { Martins; } \\
\text { Sforsin; Pinto }\end{array}$ & $\begin{array}{l}\text { Estudo de viabilização e proposta de implementação do sistema } \\
\text { kanban em uma central de atendimento por dose individualizada } \\
\text { Qualitativo, observacional descritivo }\end{array}$ & $\begin{array}{l}2017 \\
\text { BRA }\end{array}$ & $\begin{array}{l}\text { Rev. Adm Saúd } \\
\text { SciELO }\end{array}$ & $\mathrm{VI}$ \\
\hline $\begin{array}{l}\text { 17. Anschau; Silva; } \\
\text { Roessler;Webster; }\end{array}$ & $\begin{array}{l}\text { Evaluation Of Clinical Governance Interventions On qualification } \\
\text { of Care And Supply Of Bed In A Large Public Hospital }\end{array}$ & $\begin{array}{l}2017 \\
\text { BRA }\end{array}$ & Sci Med. LILACS & III \\
\hline
\end{tabular}

Klafke; Ferreira; Mers- Intervencionista de campo, quantitativo seshmidt; Fernandes

\begin{tabular}{|c|c|c|c|c|}
\hline $\begin{array}{l}\text { 18. Hitti ; El-Eid; } \\
\text { Tamim; Saleh;Saliba } \\
\text { Naffaa }\end{array}$ & $\begin{array}{l}\text { Improving Emergency Department Radiology Transportation Time: } \\
\text { a successful implementation of Lean Methodology } \\
\text { Intervencionista de campo, quantitativo }\end{array}$ & $\begin{array}{l}2017 \\
\text { LIB } \\
\text { GBR }\end{array}$ & $\begin{array}{l}\text { BMC Health } \\
\text { Services } \\
\text { PUBMED }\end{array}$ & III \\
\hline $\begin{array}{l}\text { 20. Massaro, I; } \\
\text { Massaro, A. }\end{array}$ & $\begin{array}{l}\text { O uso do Kanban na Gestão do Cuidado: superando limites } \\
\text { Descritivo, qualitativo }\end{array}$ & $\begin{array}{l}2017 \\
\text { BRA }\end{array}$ & $\begin{array}{l}\text { Rev. Adm. Saúde } \\
\text { SciELO }\end{array}$ & VI \\
\hline $\begin{array}{l}\text { 21. Sánchez; } \\
\text { Flores; } \\
\text { De La Parra; } \\
\text { Arroyo }\end{array}$ & $\begin{array}{l}\text { Improvement in the time of attention to patients in a gyneco- } \\
\text {-obstetrics emergency unit by means of the application of lean } \\
\text { manufacturing } \\
\text { Observacional, tipo estudo de caso }\end{array}$ & $\begin{array}{l}2016 \\
\text { COL }\end{array}$ & $\begin{array}{l}\text { Revista Lasallista } \\
\text { Investigación } \\
\text { SciELO }\end{array}$ & VI \\
\hline 22. Mitka & $\begin{array}{l}\text { Application of Kanban system on a hospital pharmacy } \\
\text { Qualitativo, estudo de caso }\end{array}$ & $\begin{array}{l}2015 \\
\text { GRE }\end{array}$ & $\begin{array}{l}\text { Hell J Nucl Med } \\
\text { PUBMED }\end{array}$ & VI \\
\hline 24. Pineda; González & $\begin{array}{l}\text { Improvement of the efficiency of a rehabilitation service using } \\
\text { Lean methodology } \\
\text { Qualitativo, descritivo }\end{array}$ & $\begin{array}{l}2015 \\
\text { ESP }\end{array}$ & $\begin{array}{l}\text { Rev Calid Assist. } \\
\text { PUBMED }\end{array}$ & VI \\
\hline $\begin{array}{l}\text { 25. Bang; Erikson; } \\
\text { Solnevik; }\end{array}$ & $\begin{array}{l}\text { The Nurse Watch: Design and Evaluation of a Smart Watch Appli- } \\
\text { cation with Vital Sign Monitoring and Checklist Reminders } \\
\text { Qualitativo, descritivo }\end{array}$ & $\begin{array}{l}2015 \\
\text { SWE } \\
\text { GRB }\end{array}$ & $\begin{array}{l}\text { AMIA } \\
\text { PUBMED }\end{array}$ & VI \\
\hline $\begin{array}{l}\text { 26. Bassuk; Washin- } \\
\text { gton }\end{array}$ & $\begin{array}{l}\text { Iterative development of visual control systems in a research } \\
\text { vivarium } \\
\text { Intervencionista de campo }\end{array}$ & $\begin{array}{l}2014 \\
\text { USA }\end{array}$ & $\begin{array}{l}\text { PLOS ONE } \\
\text { PUBMED }\end{array}$ & III \\
\hline
\end{tabular}

tidisciplinares de tomarem conhecimento imediato do status do paciente, adotando as medidas necessárias para mitigar falhas e solucionar pendências $^{17,19-20,25}$. O subsistema Kanban facilitou a sistematização dos processos hospitalares, gerando fluxos de atendimento eficazes, reduzindo erros e aumentando a segurança do paciente ${ }^{13}$.

A ferramenta foi utilizada em diversas versões: informatizada através de painéis, rede internas de computadores $^{17-18,20}$ ou via smart phone $^{25}$; digitalizada através de um modelo experimental de nurse watch, e, de forma mais "tradicional", através de quadros, placas ou cartões visuais, instalados em postos de enfermagem 
ou salas multiprofissionais ${ }^{19-20,24,26}$.

Um sistema de controle por placa

de "works-in-process" (quadro

de tarefas a realizar) aplicado no controle de qualidade de cobaias, comprovou que a introdução deste subsistema contribuiu para melhoria contínua de desempenho, otimizando as pesquisas de desenvolvimento de fármacos. A participação de uma equipe comprometida e consciente foi destacada como importante para os sucessos conseguidos. Houve redução de custos. A viabilidade de sustentação do sistema de gestão da pesquisa foi comprovada ${ }^{26}$.

O Kanban é uma ferramenta tão versátil que pôde ser trabalhada associada a outras ferramentas da metodologia Lean como 5S, 2P, Seis Sigma, PDCA, etc. ${ }^{18,24-}$

${ }^{26}$. Também foi associada com ferramen-

\section{Quadro 3: Objetivos, resultados e área temática dos artigos analisados}

\section{Referência}

Objetivo(s)

14.Medir a eficácia do e-kanban no gerenciamento de leitos em um hospital privado, acreditado.

15. Abordar problemas no fluxo de pacientes, identificando as causas do longo tempo de espera, sob a luz da metodologia Lean, em um ambulatório de transplante de fígado de um hospital público de ensino e pesquisa

16. Realizar diagnóstico situacional de uma central de atendimento por dose individualizada e propor Kanban para pedidos de medicamentos à logística

17. Descrever resultados alcançados em indicadores de desempenho hospitalar e na oferta de leitos de emergência de curta permanência, após aplicação da Gestão da Clínica.

18. Determinar a eficácia do uso de métodos de gestão Lean na melhoria dos tempos de transporte do Departamento de Emergência para radiografia simples

19. Descrever a implementação da metodologia kanban associada ao protocolo "Hospital Admission Risk Profile" na gestão de leitos e fluxo de pacientes nas emergências de um Hospital de Brasília.

\section{Promover o entendimento do uso do kanban como capaz de otimizar o tempo de permanência e contribuir para melhor fluxo do paciente no âmbito hospitalar}

21. Desenvolver proposta de melhoria nos tempos de atendimento através de ferramentas da metodologia Lean em uma unidade de emergência gineco obstetrica, testando a aplicabilidade da metodologia no setor saúde colombiano.

22. Redução de lojas administradas pela farmácia; melhoria do modo de operação. Soluções para espaço de armazenamento inadequado, atraso no atendimento a pacientes ou clínicas e expiração de várias formulações farmacêuticas, armazenadas por muito tempo.

\section{Resultado(s)}

Área

Temática

A ferramenta eletrônica baseada na metodologia Kanban, acessada via web por uma equipe de gerenciamento de leitos, se demonstrou eficaz na redução do tempo de permanência hospitalar dos pacientes.

Medidas corretivas adotadas: alterar o padrão de agendamento, criar um fluxograma e um guia visual Kanban para estudantes de medicina. Uma mudança no software de agendamento de consultas médicas colaborou na redução do tempo e no deslocamento do paciente. 0 tempo de espera foi reduzido em 4,5 horas.

O Kanban provou ser uma ferramenta que facilita na gestão dos processos logísticos. Porém, para ter um bom funcionamento, é necessário realizar a sensibilização de toda a equipe de colaboradores.

Fluxo de

Pacientes

Fluxo de

Pacientes

Aplicou-se Kanban no monitoramento do tempo médio de permanência. Após introdução da Gestão da Clínica, as internações aumentaram 10\% e as altas para o domicílio 101,9 em relação ao ano anterior. 0 tempo médio de permanência caiu de 7,2 para 6,6 dias, com taxa de mortalidade de 3,5 para $0,7(p<0,05)$.

A intervenção resultou em diminuição estatisticamente significativa no transporte médio radiográfico (TAT) (média \pm DP: 9,87 min versus 22,89 $\min$ ); onde $71,6 \%$ dos pacientes no período pós-intervenção tiveram TAT de transporte $\leq 10$ min, contra 32,3\% no período pré-intervenção. 0 tempo de intervenção dos pacientes também foi reduzido.

0 Hospital teve condições de abrigar o projeto Kanban, atingindo os objetivos de reduzir o tempo de espera, as filas e o tempo de internação de seus pacientes.

O Kanban só será eficaz quando manejado por uma equipe de excelência do cuidado, para qual seu emprego faça sentido. Do contrário, será somente sinalizador visual, burocrático e inconsistente, apenas para ativação de processos.

A proposta foi avaliada através de simulações baseadas no software Arena. Houve melhorias nos tempos de atenção até $56 \%$. 0 estudo evidenciou a aplicabilidade das principais ferramentas Lean. 0 uso do Kanban permitiu estabelecer os processos que precisam ser melhorados ou eliminados para diminuir os tempos de atendimento ao paciente.

0 Kanban pode favorecer a aquisição de medicamentos para estoque, por análise da necessidade de estoque, dos preços das ações dos itens e da demanda. Foram estimadas economias significativas de custos e Estoque de Medicamento vantagens operacionais futuramente. Análise, projeto e aplicação de um sistema que suporte o método de aquisição proposto foi um desafio apontado.
Estoque de

Medicamento

Quali-

dade de assistência

à saúde

Quali-

dade de assistência à saúde

Qualidade de assistência à saúde

Qualiassistência à saúde

Fluxo de Paciente dade de 
23.Comparar a aplicação dos modelos de qualidade total utilizados em processos de um serviço de saúde, a casos de uso da metodologia Lean relatadas na literatura e de uma outra instituição que á aplicou esse modelo

\section{Avaliar se a aplicação da metodologia Lean para um serviço de reabilitação reduz o desperdício e os custos com materiais e o aumenta o tempo dispensado ao atendimento ao paciente}

25. Descrever um sistema de relógio inteligente, desenvolvido para gestão da assistência de enfermagem, em colaboração com a equipe de enfermeiras de uma UTI sueca,

26. Testar a hipótese que a reintrodução da metodologia de melhoria contínua, aplicada na gestão de um hospital infantil de Seatlle, facilitaria a adesão dos profissionais do biotério à esta nova gestão, para desenvolvimento e sustentabilidade do setor.
Na unidade na qual Lean foi adotada, e Kanban aplicado na dose individualizada da farmácia, as falhas no sistema de distribuição de medicação quimioterápico ficaram perto de zero. Erros foram identificados e mitigados imediatamente. 0 serviço de enfermagem foi elencado como o indicador para qualidade.

Reduções de 43 do custo do material e $19 \%$ do consumo por cliente tradado. Aumento de $7 \%$ do tempo de atendimento ao cliente por profissional. Valorização do check list de $50 \%$ para $77 \%$. Aplicação do Kanban na área de controle, aquisição e distribuição de material reduziu significativamente o custo financeiro.

0 quadro Kanban, desenvolvido para visualização na tela multitoque do relógio inteligente, permitiu monitoramento de sinais em tempo real, alarme de limites e lembretes de tarefas, fornecendo uma visão geral das tarefas concluídas e futuras.

Redução de $17 \%$ da frequência de mudança de gaiolas proporcionou tempo adicional aos funcionários. As métricas das lavagens das gaiolas demonstraram uma melhoria no fluxo contínuo. 0 sistema de empacotamento de lote e fila tradicional foi substituído (adoção do Kanban biológico). Hipótese confirmada.
Estoque de Medicamento

\section{Quali-} dade de assistência à saúde

Quali-

dade de assistência à saúde

\section{Quali-}

dade de assistência à saúde tas de gestão clínica (projeto terapêutico singular, equipe gerencial, equipe multidisciplinar) ${ }^{17,19-20}$. Em ambos os casos demonstrou sua capacidade de flexibilidade, dinamismo e adaptação aos diversos setores da instituição na qual é adotada $^{17-20,24-25}$.

\section{Fluxo de pacientes}

O sistema Kanban é uma ferramenta que está sendo adaptada, no Brasil e no mundo, à gestão de leitos de urgência e emergência ${ }^{6,21}$, de leitos hospitalares ${ }^{14}$ e do tempo de atendimento ambulatorial $^{15}$. O gerenciamento de leitos de internação, auxiliado pela aplicação de uma ferramenta eletrônica acessada via web (e-kanban) que estratificava o tempo de internação por cores, possibilitou monitoramento e avalições sistemáticas, por parte da equipe multiprofissional, dos pacientes internados em um hospital da Suécia, avaliando inclusive os riscos sociais e os riscos clínicos. A ferramenta e-kanban, acessada de forma contínua e precisa por tablet, celulares ou computadores, permitiu a redução do tempo geral de internação, aumentando a rotatividade de leito sem reduzir a taxa de ocupação e com aumento de ganho econômico, a médio e longo prazo, para instituição. Houve, por fim, uma diminuição no tempo de espera de internação das emergências ${ }^{14}$.

A superlotação em serviços de emergência é um "fenômeno" contemporâneo global, impactando fortemente sobre a gestão clínica e a qualidade assistencial $^{28}$. Em um estudo desenvolvido na Colômbia, o uso do Kanban, associados a outras ferramentas Lean, facilitou a identificação dos processos que precisavam ser melhorados ou eliminados, para que se alcançasse a diminuição de tempos de atendimentos de pacientes, determinado por lei local. O estudo enfatizou a necessidade de formação de equipe multiprofissional, bem como sua capacitação para utilização das ferramentas disponíveis, em especial os softwares desenvolvidos para que informações sobre os pacientes fossem disponibilizadas em tempo real. Na posse destas informações os profissionais puderam tomar decisões a respeito da terapêutica, reduzindo o tempo de permanência na unidade ${ }^{21}$.

\section{Estoque de medicamentos}

Os hospitais são organizações que precisam gerenciar seus custos, pois possuem variadas frentes para distribui- ção dos recursos financeiros. A terapia medicamentosa é uma escolha de assistência que movimenta grande parte destes recursos e, se mal gerido, pode desestabilizar esta organização ${ }^{22}$. No organograma hospitalar, o serviço de farmácia é o responsável pela viabilização do controle e distribuição destes medicamentos, objetivando o uso racional destes produtos para a saúde. A Organização Pan-Americana de Saúde recomenda a racionalização da distribuição de medicamentos para minimizar erros, diminuir custos, com o objetivo máximo e aumentar a segurança do paciente ${ }^{16}$.

O sistema de distribuição unitária foi um recurso implantado no gerenciamento dos medicamentos. Atender a necessidade individual de cada paciente agregou qualidade e segurança a etapa de provisão, mas trouxe custos iniciais importantes para implantação ${ }^{16}$. Como a ferramenta kanban carreia em seu escopo a filosofia da organização dos processos com atendimento em menores prazos e redução dos custos, mostra-se congruente na sistematização do fluxo da farmácia. Contudo, para obter bons resultados, é necessário que a equipe seja treinada e motivada ${ }^{16}$.

Esta ferramenta de gestão enxuta 
possibilitou um controle dos estoques hospitalares, através do conhecimento das necessidades reais de consumo. Cartões de identificação destacando visualmente necessidades de estoque, compra, reposição, calculados com precisão reduziram a necessidade de espaço de armazenamento, atenderam mais rapidamente aos pacientes, sem desperdício financeiro. Erros nos processos de farmácia puderam ser reparados antes da destinação ${ }^{22}$.

O kanban também foi aplicado na identificação visual de procedimentos, com o intuito de minimizar dano direto ao paciente na fase de administração de quimioterápicos. Para tal foi aplicado não somente na dispensação e distribuição dos medicamentos pelo serviço de farmácia, mas também nas etapas de prescrição médica e na administração de medicamentos realizada pela equipe de enfermagem ${ }^{23}$.

A aplicação do modelo Lean Manufactoring propiciou o trabalho em equipe por agregar um pensamento sistêmico, onde todos elementos participam dos processos, através de desburocratização, e eliminando desperdícios e erros ${ }^{1}$.

\section{CONCLUSÃO}

Este estudo de revisão literatura sobre o uso da ferramenta Kanban na ges- tão de serviços de saúde, evidenciou uma produção mundial pequena, com destaque para estudos realizados no Brasil. Apontou a utilização da ferramenta em três grandes áreas: estoque de medicamento, fluxo de paciente e qualidade de assistência a saúde, refletindo positivamente na qualidade hospitalar, com diminuição de custos financeiros. Foi enfatizado que estes resultados dependem do envolvimento de equipes multiprofissionais capacitadas e motivadas, e de gestores comprometidos com a melhoria contínua dos processos para obtenção de uma assistência com mais qualidade e segurança.

\section{Referências}

1. Godinho Filho M, Fernandes F. Manufatura enxuta: uma revisão que classifica e analisa os trabalhos apontando perspectivas de pesquisas futuras. Gest Prod. 2004;11(1):1-19.

2. Oliani LH, Paschoalino WJ, Oliveira W. Ferramenta de melhoria contínua Kaizen. UNAR. 2016;12(1):57-67.

3. Periard G. Como o Kanban pode ajudar sua empesa a reduzir custos. 2015 [acesso em 05 out 2018]. Disponível em: http://www.sobreadministracao.com/como-o-kanban-pode-ajudar-sua-empresa-a-reduzir-custos/. 4. Toussaint J, Gerard RA, Adams E. Uma transformação na saúde: como reduzir custos e oferecer um atendimento inovador. Porto Alegre: Bookman; 2012.

5. Costa MSM, Oliveira CH, Paiva EJ, Neves SM, Ramos MD. Aplicação dos conceitos de takt time e tempo de ciclo para o cálculo da eficiência nos processos de um pronto atendimento hospitalar. In: XXXVII Encontro Nacional de Engenharia de Produção. 2017 out 10-13; Joinville, Brasil. Joinville: Centro de Convenções e Exposições Expoville; 2017.

6. Negri SM, Campos MD. 0 uso da ferramenta Kanban para o controle da permanência dos usuários SUS. 2012 [acesso em 13 out 2015]. Disponível em: www.convibra.com.br/upload/ paper/2012/29295191.pdf.

7. Heisler PA. Aplicação da Metodologia Kanban como ferramenta adaptada para gestão de leitos na emergência. Rio de Janeiro. Trabalho de Conclusão de Curso [Especialização em Informação Científica e Tecnologia em Saúde] - Instituto de Comunicação e Informação Científica e Tecnologia em Saúde, Fundação Oswaldo Cruz; 2012.

8. Petry D. Análise de implantação do kanban em hospitais do programa SOS emergências. Palmas. Dissertação [Mestrado Profissional em Saúde Coletiva] - Universidade Federal da Bahia; 2016.

9. Chagas F, Braga, SAG, Lustosa N, Siqueira RM, Ribeiro SG. Implantação do painel de indicadores assistenciais como ferramenta de gestão e gerenciamento do cuidado em um hospital universitário. In: Anais do I Congresso Norte-Nordeste de Tecnologias em Saúde. 2018 dez 05-08; Teresina, Brasil. Teresina: Universidade Federal do Piauí; 2018.

10. Ministério da Saúde (BR). Portaria $n^{\circ} .1 .559,01$ ago 2008. Institui a Política Nacional de Regulação do Sistema Único de Saúde - SUS. Diário Oficial da União 02 ago 2008; Seção 1.

11. Mattos CM. Aplicação do Kanban ao processo de internação de uma unidade hospitalar pediátrica. Niterói. Dissertação [Mestrado Profissional em Enfermagem Assistencial] - Escola de Enfermagem Aurora de Afonso Costa, Universidade Federal Fluminense; 2016.

12. Mendes KDS, Silveira RCCP, Galvão CM. Revisão integrativa: método de pesquisa para a incorporação de evidências na saúde e na enferma- gem. Texto Contexto Enferm. 2008:17(4):758-64.

13. Galvão M. Níveis de evidência. Acta Paul Enferm. 2006;19(2):V. Editorial.

14. Rocha HAL, Santos AKLDC, Alcântara ACC, Lima CSSDC, Rocha SGMO, Cardoso RM, Cremonin JR. Bed management team with Kanban web-based application. Int J Qual Health Care. 2018;30(9):708-14.

15. Lot LT, Sarantopoulos A, Min LL, Perales SR, Boin IFSF, Ataide EC. Using Lean tools to reduce patient waiting time. Leadersh Health Serv (Bradf Engl). 2018;31(3):343-51.

16. Fuccia IR, Matsufugi MT, Martins MC, Sforsin ACP, Pinto VB. Estudo de viabilização e proposta de implementação do sistema Kanban em uma central de atendimento por dose individualizada. Rev Adm Saúde. 2017;17(67):1-29.

17. Anschau F, Webster J, Roessler $\mathrm{N}$ et al. Avaliação de intervenções de Gestão da Clínica na qualificação do cuidado e na oferta de leitos em um hospital público de grande porte. Sci Med. 2017;27(2):1-7.

18. Hitti EA, El-Eid GR, Tamim H, Saleh R, Saliba M, Naffaa L. Improving Emergency Department radiology transportation time: a successful implementation of lean methodology. BMC Health Serv Res. 2017;17(1):625.

19. Sousa PR, Muricy MS, Simeão EP, Lima ES, Braga BC. Gestão do fluxo de pacientes em internações relacionadas ao pronto-socorro: aplicação da metodologia Kanban. RAHIS. 2017;14(1):1-18.

20. Massaro ISC, Massaro A. O Uso do Kanban na gestão do cuidado: superando limites. Rev Adm Saúde. 2017;17(66):1-8.

21. Sanchez PM, Flores JM, De La Parra PN, Arroyo JC. Improvement in the time of attention to patients in a gyneco-obstetrics emergency unit by means of the application of lean manufacturing. Rev Lasallista Investig. 2016;13(2):46-56

22. Mitka E. Application of Kanban System on a hospital pharmacy. Hell J Nucl Med. 2015;18 Suppl 1:4-10.

23. Eiro NY, Torres-Junior AS. Estudo comparativo das formas de apropriação dos modelos da Qualidade Total e Lean Production nos serviços de saúde. Rev Latino-Am Enfermagem. 2015;23(5):846-54.

24. Pineda Dávila S, Tinoco González J. Improvement of the efficiency of a rehabilitation service using Lean Healthcare Methodology. Rev Calid Asist. 2015;30(4):162-5.

25. Bang M, Solnevik K, Eriksson H. The nurse watch: design and evaluation of a smart watch application with vital sign monitoring and checklist reminders. AMIA Annu Symp Proc. 2015;2015:314-9.

26. Bassuk JA, Washington IM. Iterative development of visual control systems in a research vivarium. PLoS One. 2014;9(4):e90076.

27. Camanho GL. Nível de evidência. Rev Bras Ortop. 2009;44(6). Editorial. 